Original paper

\title{
Establishing paediatric diagnostic reference levels using reference curves - A feasibility study including conventional and CT examinations
}

\author{
Anja Almén ${ }^{a, b, *}$, Jónína Guðjónsdóttir ${ }^{c, d}$, Nils Heimland ${ }^{\mathrm{e}}$, Britta Højgaard ${ }^{\mathrm{f}}$, \\ Hanne Waltenburg ${ }^{\mathrm{f}}$, Anders Widmark ${ }^{\mathrm{e}, \mathrm{g}}$ \\ ${ }^{a}$ Medical Radiation Physics, Department of Translational Medicine, Lund University, Malmö, Sweden \\ ${ }^{\mathrm{b}}$ Department of Radiation Protection, Swedish Radiation Safety Authority, Stockholm, Sweden \\ ${ }^{\mathrm{c}}$ Icelandic Radiation Safety Authority, Reykjavik, Iceland \\ d Department of Radiography, Faculty of Medicine, University of Iceland, Reykjavik, Iceland \\ ${ }^{\mathrm{e}}$ Department of Radiation Protection and Measurement Services, Norwegian Radiation and Nuclear Safety Authority, Barum, Norway \\ ${ }^{\mathrm{f}}$ Radiation Protection, Danish Health Authority, Copenhagen, Denmark \\ ${ }^{\mathrm{g}}$ Department of Health Sciences, Norwegian University of Science and Technology, Gjovik, Norway
}

\section{A R T I C L E I N F O}

\section{Keywords:}

Diagnostic reference levels

Paediatric radiology

CT

Conventional $\mathrm{x}$-ray examinations

\begin{abstract}
A B S T R A C T
Purpose: To derive Regional Diagnostic Reference Levels (RDRL) for paediatric conventional and CT examinations using weight-based DRL curves and compare the outcome with DRL derived using the weight groups. Methods: Data from 1722 examinations performed at 29 hospitals in four countries were included. DRL was derived for four conventional x-ray (chest, abdomen, pelvis, hips/joints) and two types of CT examinations (thorax, abdomen). DRL curves were derived using an exponential fit to the data using weight as an independent variable and the respective radiation dose indices $\left(\mathrm{P}_{\mathrm{KA}}, \mathrm{CTDI}_{\mathrm{vol}}, \mathrm{DLP}\right)$ as dependent variables. DRL was also derived for weight groups for comparison. The result was compared with national diagnostic reference level (NDRL) curves.

Results: The derived curves show similarities with the NDRL curves available and corresponded sufficiently well with DRL for weight groups using the same data set, if sufficient number of data was available.

Conclusions: We conclude that weight-based DRL curves are a feasible approach and could be used together with DRL for weight groups. The main advantage of DRL curves is its application in the clinic. When the examination frequency is low, time to collect enough data to establish typical values for one or several weight groups may be unreasonably long. The curve provides the means to compare dose level faster and with fewer data points.
\end{abstract}

\section{Introduction}

Reference levels have been introduced and recommended to facilitate radiological protection [1]. These recommendations have been adopted in international safety standards [2] as well as in the European legal system [3]. Regarding medical exposures and particularly diagnostic radiology, reference levels have been introduced as diagnostic reference levels (DRL) [4]. DRL - local (LDRL), national (NDRL) or regional (RDRL) - is defined for a specific radiological examination regarding a typical patient using a well-defined modality-specific radiation quantity. The typical dose for the typical patient is determined in the clinic and then compared with a DRL.

The prerequisites for dose data collections and thus setting and applying DRL in hospitals have improved in recent years. Modern X-ray equipment provides output of standardised radiation quantity values available in the clinic [5-7] and dose management systems aid with the collection and analysis of the data [8]. For conventional and fluoroscopy examinations, air kerma area product $\left(\mathrm{P}_{\mathrm{KA}}\right)$ is available and for computed tomography (CT) the computed tomography dose index $\left(\mathrm{CTDI}_{\mathrm{vol}}\right)$ and the dose length product (DLP) are available. These quantities do not directly give radiation dose to patients but serve as a substitute and are readily available and thus used in the system of DRLs. A guidance document in the Technical Report Series of the IAEA specify the quantities and required accuracy [6]. However, specifying a typical patient remains a challenge.

The specification of a typical patient is needed to derive DRL. For

\footnotetext{
* Corresponding author at: Medical Radiation Physics, Department of Translational Medicine, Lund University, Malmö, Sweden.

E-mail address: anja.almen@med.lu.se (A. Almén).
} 
Table 1

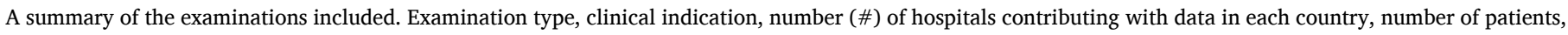
weight (median, mean, 1st quartile (q1) and 3rd quartile (q3)), $\mathrm{P}_{\mathrm{KA}}$ (median, mean, 1st quartile (q1) and 3rd quartile (q3)).

\begin{tabular}{|c|c|c|c|c|c|}
\hline Examination & Clinical indication & $\begin{array}{l}\text { \# hospitals (DK, IS, NO, } \\
\text { SE) }\end{array}$ & $\begin{array}{l}\text { \# Patients (DK, IS, NO, } \\
\text { SE) }\end{array}$ & $\begin{array}{l}\text { Weight, kg median (mean) q1, } \\
\text { q3 }\end{array}$ & $\begin{array}{l}\mathrm{P}_{\mathrm{KA}}, \mathrm{Gycm}^{2} \text { median (mean) q1, } \\
\mathrm{q} 3\end{array}$ \\
\hline \multirow[t]{2}{*}{ chest erect } & \multirow[t]{2}{*}{ general indications } & \multirow[t]{2}{*}{$17(7,2,4,4)$} & \multirow[t]{2}{*}{$361(243,11,69,38)$} & $32(36)$ & $0.025(0.043)$ \\
\hline & & & & 23,48 & $0.012,0.049$ \\
\hline \multirow[t]{2}{*}{ chest supine } & \multirow[t]{2}{*}{ general indications } & \multirow[t]{2}{*}{$11(5,1,1,4)$} & \multirow[t]{2}{*}{$138(27,62,6,43)$} & $10(10)$ & $0.030(0.032)$ \\
\hline & & & & 8,12 & $0.015,0.040$ \\
\hline \multirow[t]{2}{*}{ abdomen } & \multirow[t]{2}{*}{ overview general indications } & \multirow[t]{2}{*}{$18(6,2,3,7)$} & \multirow[t]{2}{*}{$346(40,18,111,177)$} & $20(23)$ & $0.090(0,258)$ \\
\hline & & & & 12,31 & $0.039,0.240$ \\
\hline \multirow[t]{2}{*}{ pelvis } & \multirow[t]{2}{*}{ fracture, skeletal questions } & \multirow[t]{2}{*}{$15(7,-, 4,4)$} & \multirow[t]{2}{*}{$286(162,-, 74,50)$} & $20(26)$ & $0.067(0.163)$ \\
\hline & & & & 13,35 & $0.023,0.194$ \\
\hline \multirow[t]{3}{*}{ hips/joints } & \multirow[t]{3}{*}{ dysplasia } & \multirow[t]{3}{*}{$14(5,-, 3,6)$} & \multirow[t]{3}{*}{$263(70,-, 57,136)$} & $10(10)$ & $0.023(0.036)$ \\
\hline & & & & 9,12 & $0.010,0.047$ \\
\hline & & & & & CTDI. mGy \\
\hline \multirow[t]{6}{*}{ CT thorax } & \multirow[t]{6}{*}{ tumour, with contrast } & \multirow[t]{6}{*}{$8(2,-, 4,2)$} & \multirow[t]{6}{*}{$121(19,-, 62,40)$} & $18(25)$ & $1.4(1.9)$ \\
\hline & & & & 12,35 & $1.0,2.2$ \\
\hline & & & & & DLP. mGycm \\
\hline & & & & & $33.5(48.4)$ \\
\hline & & & & & $21.0,58.0$ \\
\hline & & & & & CTDI. mGy \\
\hline \multirow[t]{5}{*}{ CT abdomen } & \multirow{5}{*}{$\begin{array}{l}\text { tumour or inflammation, no } \\
\text { contrast }\end{array}$} & \multirow[t]{5}{*}{$14(4,-, 5,5)$} & \multirow[t]{5}{*}{$207(30,-, 30,147)$} & $38(39)$ & $2.7(3.0)$ \\
\hline & & & & 27,50 & $1.8,3.7$ \\
\hline & & & & & DLP. mGycm \\
\hline & & & & & $113.3(129.2)$ \\
\hline & & & & & $72.8,167.3$ \\
\hline
\end{tabular}

adults, this parameter is usually weight and a target average weight for the group of patients for which the DRL are determined is therefore defined. The average weight of $70 \pm 3 \mathrm{~kg}$ has been used as a criterion specifying the typical adult patient [4]. Following this, the typical dose is derived also for this typical patient. However, it has been found more difficult to define a typical patient for children and this may be one reason the system of DRLs is seldom applied in paediatric radiology [9].

A commonly used parameter characterising a child is age. DRL and thus the typical dose for the paediatric patients have been derived for age groups [10-12]. The main reason for this may be that age is a readily available parameter. Anatomy and pathology are also age-dependent for children, and certain radiological examinations may be more common for a specific age group. However, the parameter that determines radiation dose to the patient is largely depending on the tissue density and thickness of the examined body part. Taking this into account, weight may be a better parameter, compared to age. Recently, the European Commission issued guidelines for European paediatric diagnostic reference levels (EDRLs) [13]. In these guidelines, five weight groups are specified and recommended. However, one other issue remains in this context. The number of examinations of children is limited and this makes it difficult to obtain data to determine DRLs. The application in the clinical setting is also more difficult, a sufficient number of data must be provided for each weight group. Due to the limited number it could be anticipated that it takes a long time for hospitals to gather enough data, if manageable at all. However, alternatives exist to the previous suggestions of collecting data for weight groups.

It has been suggested that a mathematical fit to the radiation dose could be used to describe the relationship between patient weight and radiation dose [14] or to define a DRL curve to be used as an alternative to DRL values regarding a typical patient [15]. The method is based on the increase of radiation dose with the size of the patient. Therefore, in radiation dose surveys equivalent diameter or weight has been used as substitute for thickness and radiation dose has been evaluated using a curve fit to dose data [16]. To our knowledge, only Denmark and Finland have previously derived curves as NDRL $[17,18]$.

The aims of this study were to (i) investigate possibilities and limitations in the development of weight-based regional diagnostic reference levels curves (RDRL curves), (ii) derive RDRL curves for conventional and computed tomography examinations and explore their usefulness and (iii) compare the method of using weight-based groups and weight-based curves.

This was an empirical study comprising data from hospitals in Denmark, Iceland, Norway and Sweden in order to explore the prerequisites to apply the concept of diagnostic reference levels using clinical data.

\section{Material and methods}

\subsection{Patient cohorts and data collected}

Data regarding both conventional x-ray examinations as well as CT examinations were collected from 31 hospitals in Denmark, Iceland, Norway and Sweden using a web-based collection system. An overview of collected data is presented in Table 1 . The examinations were chosen based on an estimate of the number of examinations carried out in the clinics, i.e. it should be possible to collect data during a reasonable time frame. The hospitals known to perform a relevant number of paediatric examinations were asked to participate. In this study, a paediatric patient was defined as being under 16 years of age. All hospitals have X-ray equipment with automatic exposure control. These are used, except in certain conventional X-ray examinations of the youngest children. We assume, based on our experience, that all hospitals to some extent adapt the exposure to the patient's size.

The hospitals were asked to report basic data for at least 10 patients for each examination type. The data were collected during 2018 and 2019. Formal ethical approval was not required as patient dose collection to determine diagnostic reference levels is performed according to national regulations. Data collected cannot be linked to an individual patient. The data reported were the radiation dose indices - $\mathrm{P}_{\mathrm{KA}}$ for radiography and DLP for CT - for a complete examination together with patient weight. For conventional radiology the number of images was also reported and for CT examinations, the number of phases and the $\mathrm{CTDI}_{\mathrm{vol}}$ (with reference to the $32-\mathrm{cm}$ phantom) from the phase with the highest value was reported. The technical parameters, such as x-ray beam quality, were not collected. The examination types were described using both anatomical area exposed and clinical indication. The examinations were anticipated to be performed using a similar technique except for conventional chest $\mathrm{x}$-rays. For this type the examination techniques differ substantially for patients of different age. When the child is able to stand or sit, they may be examined in an erect PA or AP 
position. Alternatively, the child is helped to be in an erect position in front of the detector (AP view). The younger patients may also be examined in the supine position (AP view) on the table-top. Neonates in intensive care may be examined in the incubator. Therefore, data were studied separately for erect and supine chest examinations and neonates examined in intensive care were not included in the study.

For each examination type, the data were summarized using descriptive statistics, calculating mean, 25th percentile (1st quartile), median (2nd quartile) and 75th percentile (3rd quartile) values for the fundamental parameters collected, in order to get an overview of the data set.

\subsection{Deriving regional diagnostic reference levels}

The basis for the method is to use a curve fit to the collected dose indices, with the dose indices as the dependent variable and a patient parameter as the independent variable. The RDRL curves were derived using data from the particular examinations assuming an exponential increase of the patient dose indices with patient weight for each examination type respectively. The technique was similar to the methods previously suggested [19]. The reason for assuming an exponential increase with weight was that the method is based on basic physics, i.e. the $\mathrm{X}$-ray beam exhibits exponential attenuation in the patient body. The attenuation depends on the tissue thickness, density, elemental composition and X-ray beam energy [20].

An exponential curve $-\mathrm{D}(\mathrm{w})=\mathrm{a} \cdot \mathrm{e}^{(\mathrm{k} \cdot \mathrm{w})}-$ was derived using Microsoft Excel. Here " $D$ " is the dose index $\left(\mathrm{P}_{\mathrm{KA}}, \mathrm{CTDI}_{\mathrm{vol}}\right.$ or DLP) collected for each patient and " $w$ " the corresponding weight of the patient. The method assumes that the exponential growth, indicated by the regression coefficient $(\mathrm{k})$, for an examination type is similar for all departments. The k-value is associated with the exponential increase of the dose indices and was derived for the different examinations. The a-value was calculated using the k-value, the weight and dose value for each patient. Thereafter, the third quartile value $\left(D_{\mathrm{q} 3}\right)$ for the "a-values" was derived. Likewise, the median value $\left(\mathrm{D}_{\mathrm{q} 2}\right)$ of the data set was derived. The latter was used to derive a curve indicating an achievable dose level (ADL). The DRL curve was defined as: $\mathrm{DRL}(\mathrm{w})=\mathrm{D}_{\mathrm{q} 3} \cdot \mathrm{e}^{(\mathrm{k} \cdot \mathrm{w})}$ and the ADL curve as: $\mathrm{ADL}(\mathrm{w})=\mathrm{D}_{\mathrm{q} 2} \cdot \mathrm{e}^{(\mathrm{k} \cdot \mathrm{w})}$. The derived mathematical constants were studied.

The RDLR curves were derived including all available data for a specific examination type. Comparisons with RDRL calculated for the data collected in this study and existing NDRL curves from Finland were done when possible [18].

For all weight groups with a total number of patients more than ten, RDRL were derived as the third quartile of all the collected data pooled together, for each weight group and examination type. Due to the small amount of data in each weight group, it was deemed not possible to derive the third quartile of medians, as recommended in the European guidelines [13]. Furthermore, one weight group was slightly modified; in our study a maximum weight of $70 \mathrm{~kg}$ was used.

\section{Results}

\subsection{Patient cohorts and descriptive data}

In total, 1722 examinations were included in this study. A summary of the data is presented in Table 1 . The largest cohorts constitute conventional erect chest examinations and abdomen examinations (about 350 examinations in each group) and the smallest cohort was CT thorax (121 examinations).

The conventional chest examinations in erect position and CT abdomen comprised the heaviest patients with an average weight of about $35 \mathrm{~kg}$. The conventional chest supine examination and hips/joints exhibited lightest group of patients; the average weight was about $10 \mathrm{~kg}$. The other three examination types included patients with an average weight of around $20 \mathrm{~kg}$. These data show a systematic difference
Table 2

The mathematical coefficients and constants for the DRL and ADL curves for each examination type. $\mathrm{D}_{\mathrm{q} 2}$ and $\mathrm{D}_{\mathrm{q} 3}$ refer to $\mathrm{ADL}$ and $\mathrm{DRL}$ respectively.

\begin{tabular}{|c|c|c|c|c|c|}
\hline DRL & $\begin{array}{l}\text { Examination } \\
\text { type }\end{array}$ & $\mathrm{k}$ & $\begin{array}{l}\mathrm{D}_{\mathrm{q} 2} \\
\left(\mathrm{Gycm}^{2}\right)\end{array}$ & $\begin{array}{l}\mathrm{D}_{\mathrm{q} 3} \\
\left(\mathrm{Gycm}^{2}\right)\end{array}$ & $\begin{array}{l}\text { Valid for } \\
\text { interval }(\mathrm{kg})\end{array}$ \\
\hline RDRL & chest, erect & 0.036 & 0.0075 & 0.0128 & $15-70$ \\
\hline RDRL & chest, supine & 0.143 & 0.0054 & 0.0078 & $5-15$ \\
\hline RDRL & abdomen & 0.056 & 0.0284 & 0.0608 & $5-70$ \\
\hline - & $\begin{array}{l}\text { abdomen, one } \\
\text { image }\end{array}$ & 0.047 & 0.0178 & 0.0349 & - \\
\hline- & $\begin{array}{l}\text { abdomen two } \\
\text { images }\end{array}$ & 0.067 & 0.0371 & 0.0708 & - \\
\hline RDRL & pelvis & 0.065 & 0.0142 & 0.0243 & $5-70$ \\
\hline - & pelvis, one image & 0.067 & 0.0112 & 0.0204 & - \\
\hline- & $\begin{array}{l}\text { pelvis two } \\
\text { images }\end{array}$ & 0.063 & 0.0154 & 0.0265 & - \\
\hline \multirow[t]{2}{*}{ RDRL } & hips/joints & 0.144 & 0.0052 & 0.0105 & $5-15$ \\
\hline & & \multicolumn{4}{|c|}{ CTDIvol, mGy } \\
\hline RDRL & CT thorax & 0.017 & 0.93 & 1.08 & $5-70$ \\
\hline \multirow[t]{2}{*}{ RDRL } & CT abdomen & 0.020 & 1.16 & 1.56 & $10-70$ \\
\hline & & \multicolumn{4}{|c|}{ DLP, mGycm } \\
\hline RDRL & CT thorax & 0.030 & 15.2 & 19.3 & $5-70$ \\
\hline RDRL & CT abdomen & 0.029 & 33.5 & 44.3 & $10-70$ \\
\hline
\end{tabular}

between weight distributions and range of weights for patients in the different examination groups.

\subsection{Regional diagnostic reference levels}

Table 2 shows the mathematical coefficients and constants for the derived curves. The appropriate weight interval for which the curve is valid is also included in the table. RDRL curve coefficients and constants were derived from all the collected data for each examination type as labelled in the first column. Additional calculations were done to further analyse subsets of the data for two conventional radiography

Table 3

The number (\#) of patients, weight (median, mean, 1st quartile (q1) and 3th quartile (q3)) and regional diagnostic reference level (RDRL) for each of the weight groups.

\begin{tabular}{|c|c|c|c|c|}
\hline $\begin{array}{l}\text { Examination/ } \\
\text { weight group }\end{array}$ & $\begin{array}{l}\# \\
\text { patients }\end{array}$ & $\begin{array}{l}\text { Weight, } \mathrm{kg} \\
\text { median (q1, q3) }\end{array}$ & $\begin{array}{l}\text { RDRL P } P_{K A} \text {, } \\
\text { Gycm }^{2}\end{array}$ & \\
\hline \multicolumn{5}{|l|}{ chest supine } \\
\hline$[5-<15 \mathrm{~kg}]$ & 128 & $10(8,12)$ & 0.040 & \\
\hline $\begin{array}{l}{[15-<30 \mathrm{~kg}]} \\
\text { chest erect }\end{array}$ & 11 & $16(16,17)$ & 0.055 & \\
\hline$[15-<30 \mathrm{~kg}]$ & 137 & $22(19,25)$ & 0.028 & \\
\hline$[30-<50 \mathrm{~kg}]$ & 131 & $36(32,43)$ & 0.050 & \\
\hline $\begin{array}{l}{[50-<70 \mathrm{~kg}]} \\
\text { abdomen }\end{array}$ & 84 & $58(53,63)$ & 0.097 & \\
\hline$[5-<15 \mathrm{~kg}]$ & 96 & $10(8,12)$ & 0.073 & \\
\hline$[15-<30 \mathrm{~kg}]$ & 134 & $21(17,25)$ & 0.237 & \\
\hline$[30-<50 \mathrm{~kg}]$ & 75 & $35(31,42)$ & 0.534 & \\
\hline \multicolumn{5}{|l|}{ pelvis } \\
\hline$[5-<15 \mathrm{~kg}]$ & 87 & $11(9,13)$ & 0.041 & \\
\hline$[15-<30 \mathrm{~kg}]$ & 104 & $20(16,24)$ & 0.130 & \\
\hline$[30-<50 \mathrm{~kg}]$ & 49 & $39(32,43)$ & 0.330 & \\
\hline$[50-<70 \mathrm{~kg}]$ & 42 & $54(51,60)$ & 0.646 & \\
\hline \multicolumn{5}{|l|}{ Hips/joints } \\
\hline$[5-<15 \mathrm{~kg}]$ & 234 & $10(9,12)$ & 0.022 & \\
\hline$[15-<30 \mathrm{~kg}]$ & 23 & $16(15,18)$ & 0.047 & \\
\hline CT thorax & & & $\begin{array}{l}\mathrm{CTDI}_{\mathrm{vol}} \text {, } \\
\mathrm{mGy}\end{array}$ & $\begin{array}{l}\text { DLP, } \\
\text { mGycm }\end{array}$ \\
\hline$[5-<15 \mathrm{~kg}]$ & 34 & $10(9,12)$ & 1.2 & 25.3 \\
\hline$[15-<30 \mathrm{~kg}]$ & 38 & $19(17,25)$ & 1.6 & 41.4 \\
\hline$[30-<50 \mathrm{~kg}]$ & 24 & $36(34,40)$ & 2.4 & 64.7 \\
\hline$[50-<70 \mathrm{~kg}]$ & 17 & $55(51,63)$ & 3.0 & 103 \\
\hline \multicolumn{5}{|l|}{ CT abdomen } \\
\hline$[15-<30 \mathrm{~kg}]$ & 60 & $24(21,27)$ & 2.6 & 91.7 \\
\hline$[30-<50 \mathrm{~kg}]$ & 82 & $40(34,45)$ & 3.4 & 150 \\
\hline$[50-<70 \mathrm{~kg}]$ & 57 & $58(53,64)$ & 5.0 & 247 \\
\hline
\end{tabular}


examination types, abdomen and pelvis, as there were systematic differences in number of images acquired. It was obvious from the data that a typical abdomen examination in Sweden consists of two images but only one in the other countries, similarly, a typical pelvis examination consists of only one image in Norway but two in the other countries. The RDLR for weight groups calculated in this study is shown in Table 3. There were relatively few patients weighing over $50-55 \mathrm{~kg}$, for the conventional chest supine and hips/joint examinations maximum weight is even lower, all patients weighted under $30 \mathrm{~kg}$.

Figs. 1-7 show the derived RDRL curves together with the derived RDRL for weight groups for all examination types. The actual median weight of the patients is used when placing this DRL for weight groups in the scatter diagram. The derived achievable dose curves (ADL curve) are also shown in the figures. Figs. 1 and 4-7 show the results from this study compared to the existing Finnish NDRL.

\section{Discussion}

The present study explored the possibility to derive RDRL curves including four countries. RDRL curves were derived for four conventional and two CT examinations. We revealed some issues when collecting data and deriving the curves. The frequency of examinations has to be included in the overall evaluation of feasibility to derive RDRL. It took substantially longer time than anticipated to collect data. The clinical indications are important but may be an issue especially when including data from different countries as language, tradition and culture differences may exist. The lack of data for patient weighing more than $50 \mathrm{~kg}$ was an issue for all examinations but CT abdomen. In this study, we did not investigate the influence of technical acquisition parameters on the dose values, as this was not the purpose of the study. However, both technical and methodological data have to be available in the optimisation process when analysing the data and preferably shared between hospitals.

The number of examinations (Table 1) for each examination type varies from 121 to 361 . Consequently, the number of cases in each weight groups were limited, especially for the heaviest weight groups. This is an issue both when using DRL curves but also and perhaps more importantly when deriving weight-based DRL. This may have an impact on the derived DRL curves because extrapolation beyond the actual range of weights is questionable. It can be argued that none of the DRLs should apply to weights over $50 \mathrm{~kg}$.
The relatively low frequency of paediatric examinations has to be taken into account when methods for DRL regarding paediatric radiology are selected. The system of diagnostic reference levels also depends on reasonably fast data collection. Many of the study limitations stems from this limited number of examinations included. These shortcomings are difficult to overcome because the number of children examined is relatively low and including clinical indications further limits the number of examinations.

The regression coefficients for the examination types (Table 2) show differences for the conventional examinations. A more detailed analysis, including the radiation beam quality parameters may be valuable to explore the differences in more detail. The number of radiographs, one or two, for pelvis examination did not affect the curve substantially. This indicates a large difference in radiation dose levels in the departments, the reason must be investigated further.

The derived DRL curves for the conventional chest examinations are substantially different for the two different examinations techniques (Fig. 1). This shows, the importance of defining the imaging technique as well as to determine the weight range to which the curves apply. The justification of the difference has to be explored further as it indicates optimisation needs. The suggested appropriate intervals for all examinations included is indicated in Table 2. The shape of the curve for abdomen and pelvis examinations is similar (Fig. 2). However the levels differ somewhat. The derived DRL curve for conventional hips/joints (Fig. 3) only applies to a limited weight range, but there is no obvious reason that the curve is not useful despite of this. The rather low number of patients for CT thorax in each weight group is the plausible reason for the difference between RDRL curves and DRL for weight groups (Figs. 4 and 5). The RDRL curve and DRL for weight groups for CT abdomen examinations are in good agreement (Figs. 6 and 7).

In this study, the DRL for weight groups correlated fairly well with the result of the RDRL curves. One could even suggest that DRL for weight groups may be used to derive a curve if the average weight for each weight group is known and the number of data in each group is sufficient. It could be advantageous to publish both DRL curves and DRL for weight groups, if possible. The weight range for which the curve is relevant should be stated when a DRL curve is given.

For comparison, the Finnish NDRL curves are included in Figs. 1 and 4-7. Note that for the conventional chest examination the Finnish curves were derived for AP/PA and LAT respectively. For comparison in this study, the two curves have been combined. The derived achievable dose

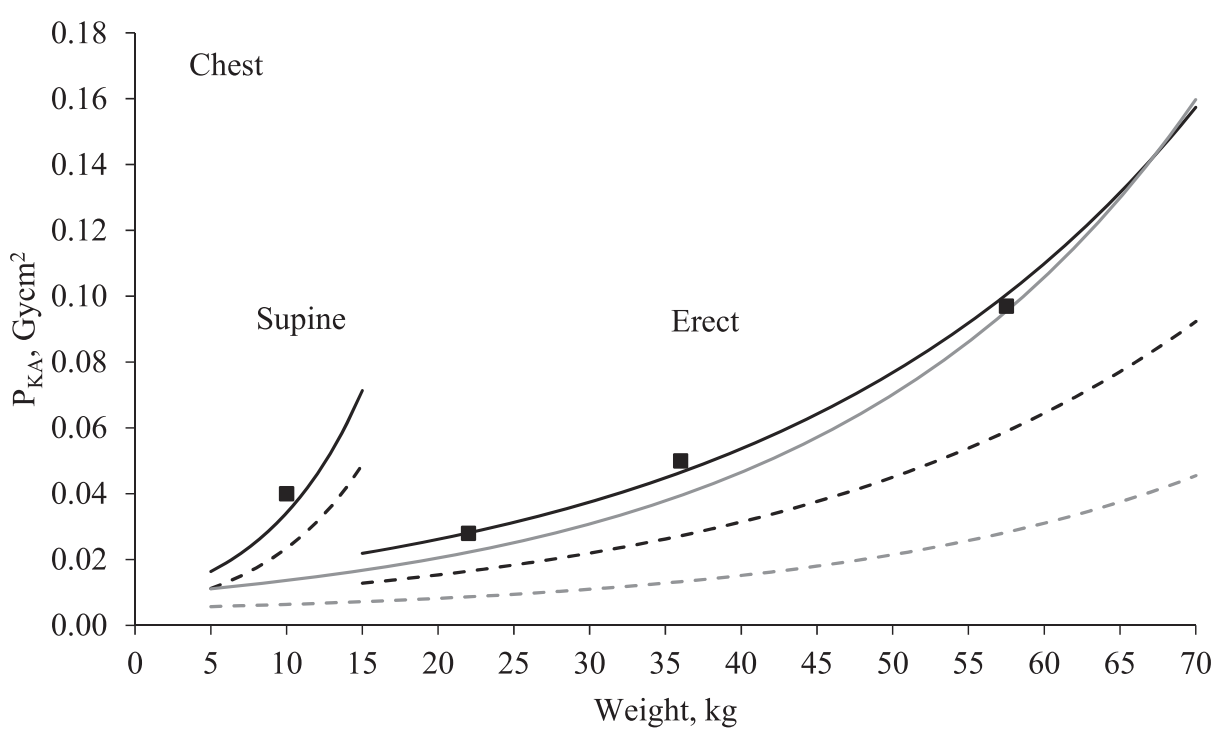

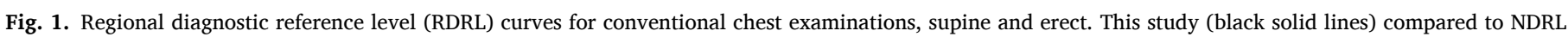

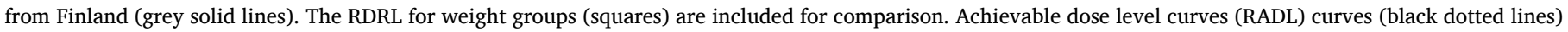
compared to NADL from Finland (grey dotted line) are included. 


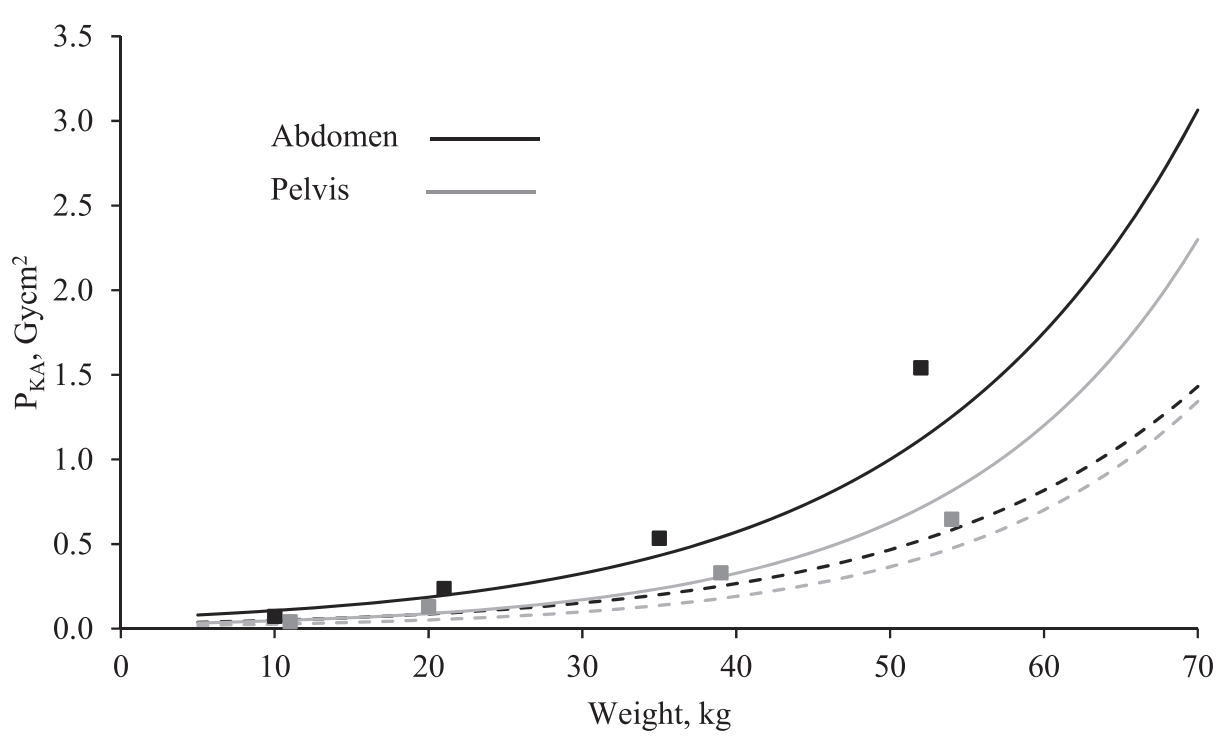

Fig. 2. Regional diagnostic reference level (RDRL) curve (solid line) and regional achievable dose level RADL curves (dotted line) for conventional abdomen (black) and pelvis (grey) examinations. The RDRL for weight groups, conventional abdomen (black squares) and pelvis (grey squares), are included for comparison.

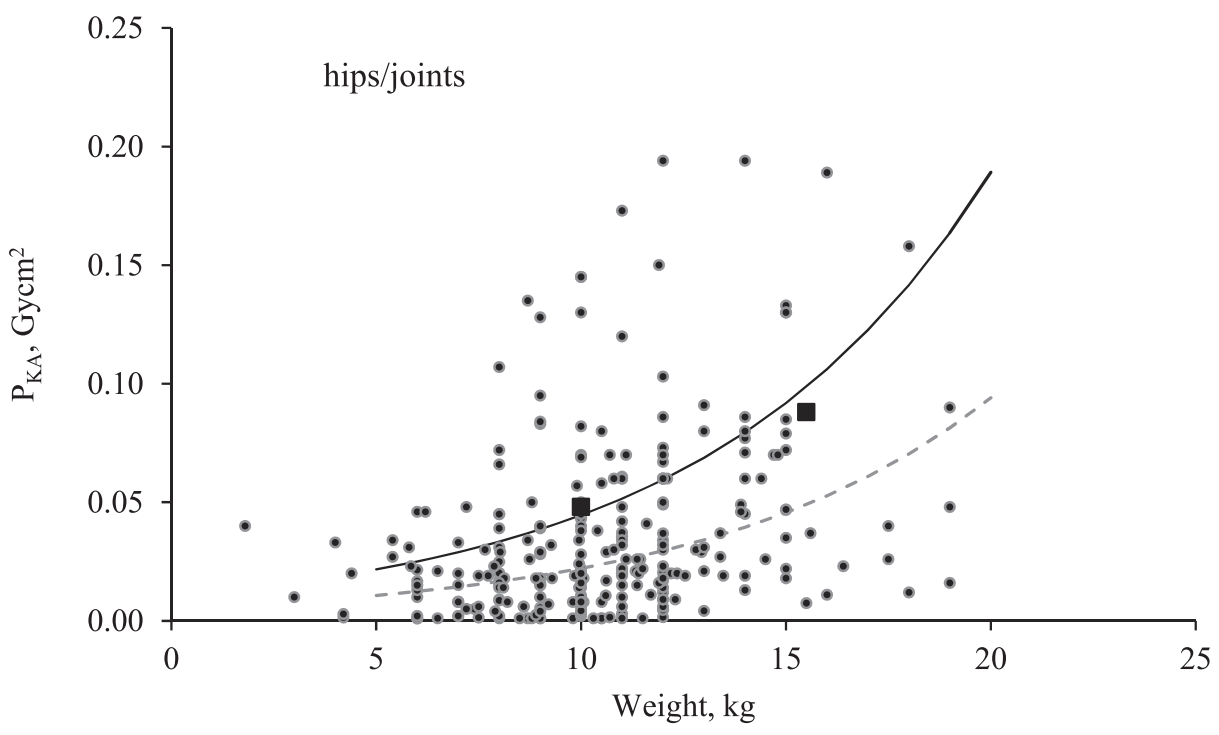

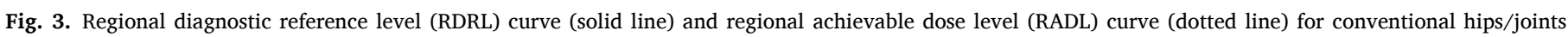
examination, all data points are included to illustrate the uneven weight distribution. The RDRL for weight groups (black squares) are included for comparison.

curves (ADL curve) are also shown in the figures. NDRLs from Finland and RDRLs derived in this study were quite similar for conventional chest examinations. However, the NDRL curves for the CT chest and CT abdomen from Finland are slightly steeper compared to the curves developed in this study. This could be due to differences in the methodology deriving the curves or that the number of patients in the higher weight groups affects the slope. However, detailed information on the exact technique and data set used in Finland were not available. The data from this study and the data on which the Finnish curves are based must be further examined to deduce the causes.

In our study, an exponential curve was used when deriving the reference curves. The regression coefficients for specific departments and a specific examination type were generally in good agreement despite the low number of data. A few outliers in this respect exist but did not affect the overall outcome given the purpose of diagnostic reference levels. A thorough investigation of data evaluation methods regarding adult CT examinations have been carried out and to some extent also supports a rather simple approach [21]. Our approach also follows basic radiation dosimetry principles as previously argued [22]. Given the rather low frequency of paediatric examinations it may be important to further explore other strategies. Other strategies are possible, applying different regression methods, a quadratic regression analysis has, to some extent, been explored [23] but the benefit in the context of DRL remains to be demonstrated. In this study, however, we did not investigate these possibilities, and this can be seen as a limitation to the study.

In this study, weight was used as an independent variable when the curves were derived. However, other variables may be used. Age has been suggested due to its availability. However, child growth does not follow a smooth mathematical equation [24] and there are large differences between countries [25]. Weight could therefore be considered a more appropriate variable to use for deriving reference curves compared with age. Alternative variables could be body mass index (BMI) or equivalent diameter [16] assuming available data for both weight and height. For CT examinations other concepts have been established in order to better take into account the actual thickness of the body part. 


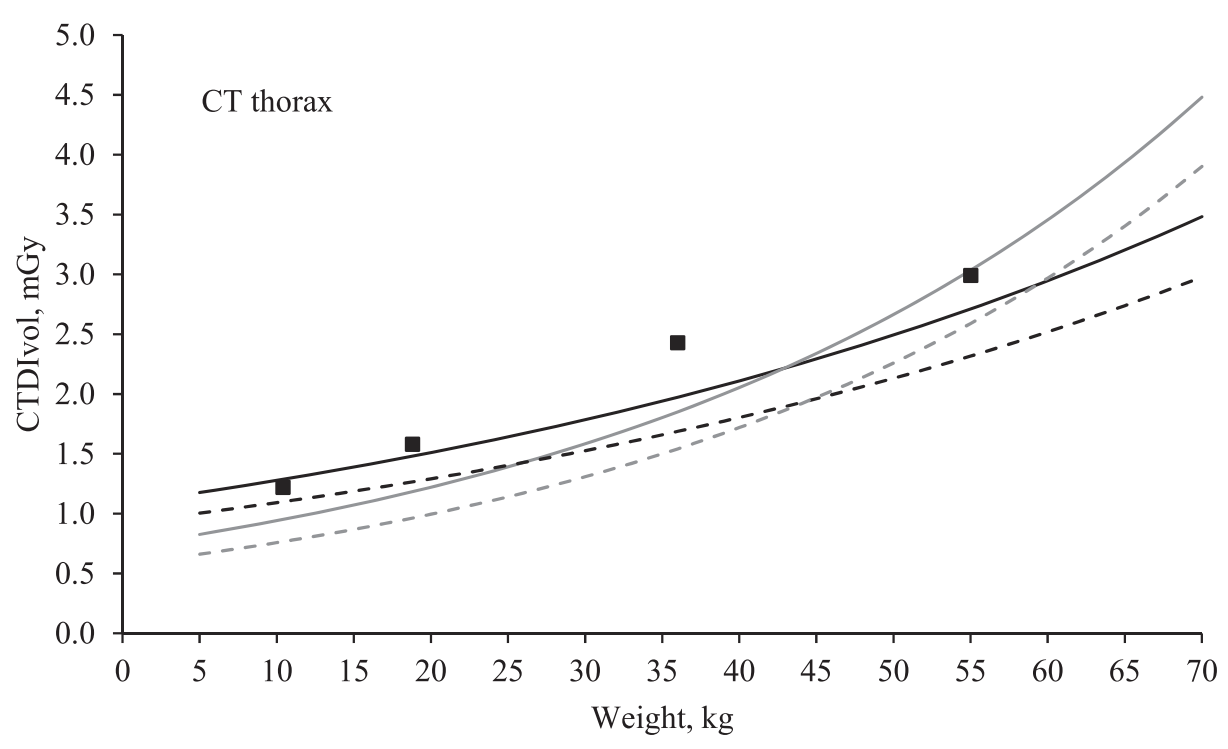

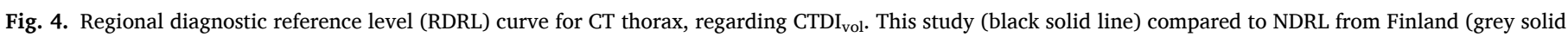

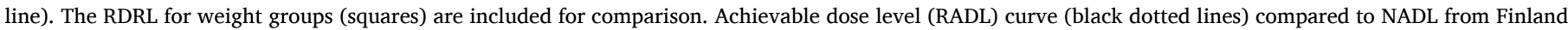
(grey dotted line) are also included.

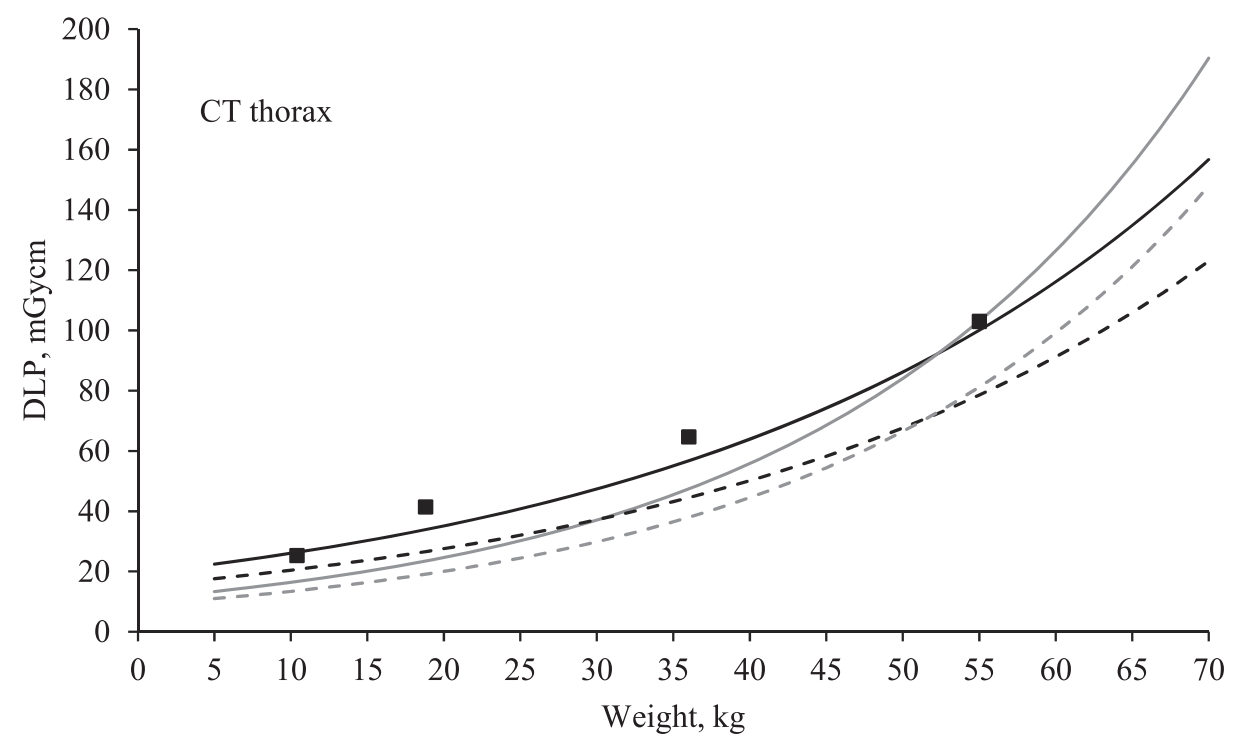

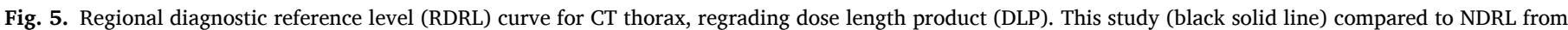

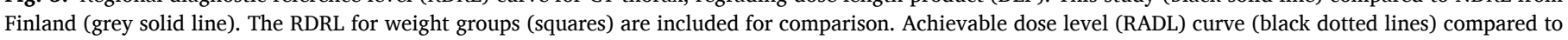
NADL from Finland (grey dotted line) are also included.

The concept of size-specific dose estimate (SSDE) includes the effective diameter based on the AP and lateral thickness of the actual patient examined [26]. The concept of water equivalent diameter (WED) derived from the size of the patients using the CT image [27] is also an alternative. Both these concepts to some extent take the size of the irradiated body part into account and are promising parameters to be included in the concept of DRL, if automatically derived and readily available in the clinic. As far as we know, similar concepts are lacking for conventional investigations.

The possible advantage of using DRL curves as a complement or replacement for using weight groups may be the application in hospitals. The amount of data from patients required to determine whether the DRL is exceeded is lower compared to collecting an appropriate number of data for a weight group. It has been suggested that data from at least ten examinations per weight group should be used to determine a typical value and this may take an unreasonably long time. By using curves, more hospitals, which perform a few paediatric examinations, can use DRL as a guide. That is, data from a small number of examinations, where the patient's weight may vary within the entire weight range to which the curve applies, can be used to compare with the established DRL curve. The clinics do not need to derive a curve but can compare the individual values with the DRL curve and evaluate whether the majority of values are above or below the curve. Thus the DRL curve may make it easier to give a quick indication of dose levels. The disadvantage is that a practical procedure for applying a curve may seem more difficult. Whether this is an actual problem needs to be investigated. Calculating the median value can be simpler and the method more intuitively understandable.

In the future, efficient databases including relevant data such as patient sizes, acquisition parameters, clinical indication and complimentary evaluation of image quality could improve the concept of diagnostic reference levels. 


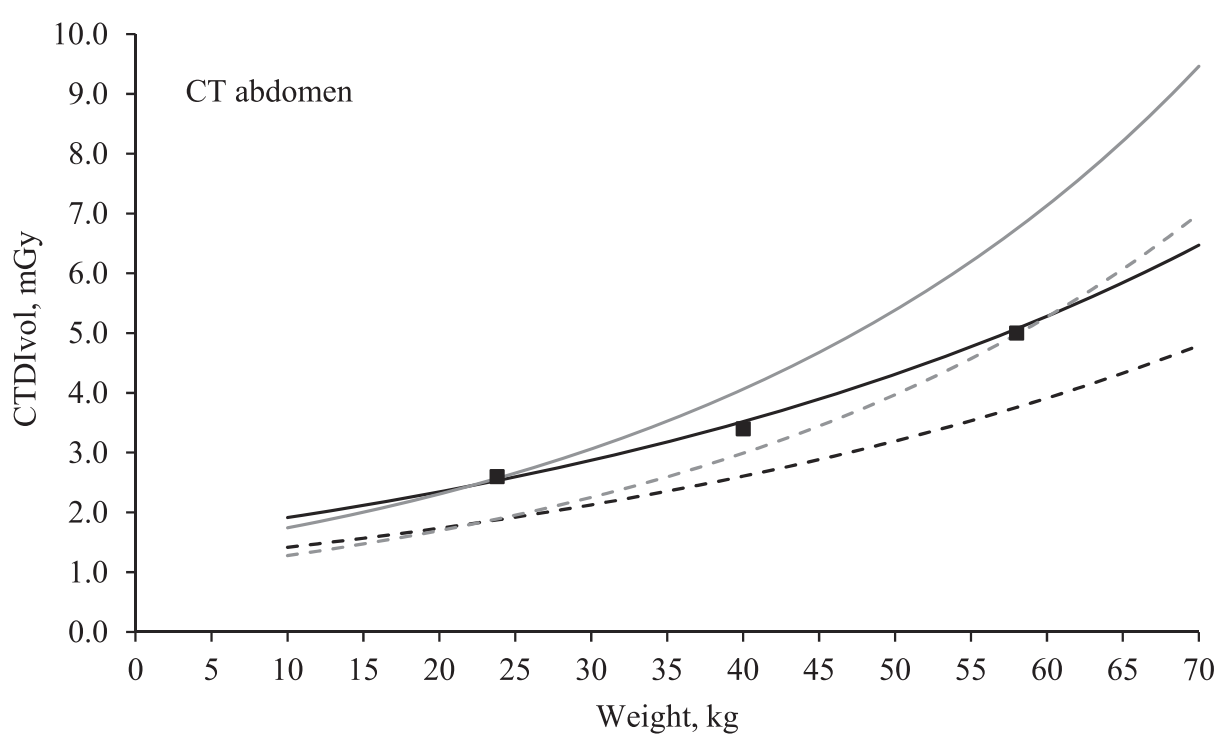

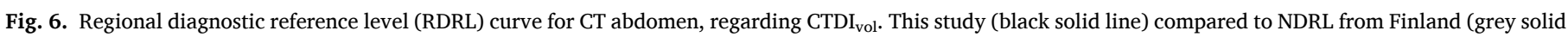

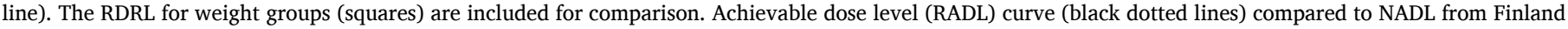
(grey dotted line) are also included.

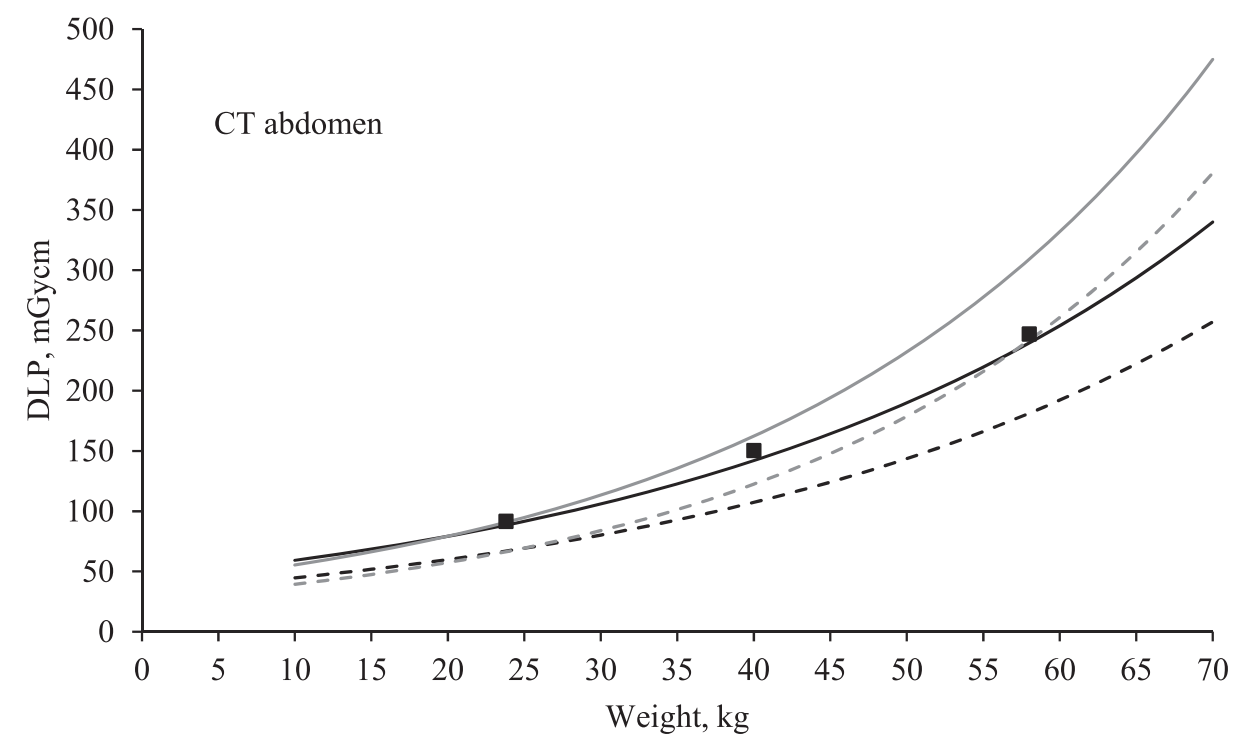

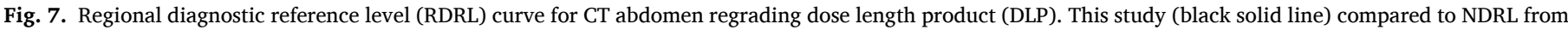

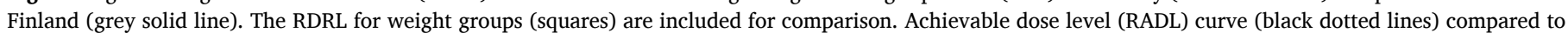
NADL from Finland (grey dotted line) are also included.

\section{Conclusions}

We conclude that weight-based DRL curves are a feasible approach and could be used as a supplement to DRL for weight groups. We also conclude that this study indicates that the different methods, using curves or weight groups, gave reasonably similar result, but the differences increased when the number of data points were few and this needs to be explored further.

The derived curves could serve the same purpose as a DRL for weight groups. That is, a dose index for one patient above the curve is not a reason for concern, but if the dose indices for the majority of the patients are above the DRL curve an investigation about the reason should be performed and if possible the dose should be reduced.

The main advantage of DRL curves is its application in the clinic. When the examination frequency is low, time to collect enough data to establish typical values for one or several weight groups may be unreasonably long. The curve provides the means to compare dose level faster and with fewer data points.

\section{Declaration of Competing Interest}

The authors declare that they have no known competing financial interests or personal relationships that could have appeared to influence the work reported in this paper.

\section{Acknowledgement}

We would like to thank all the hospitals participating in the study. 


\section{References}

[1] ICRP. ICRP Publication 103. The 2007 recommendations of the International Commission on Radiological Protection. Ann. ICRP 2007;37:1-332.

[2] International Atomic Energy Agency. Radiation protection and safety of radiation sources: international basic safety standards. IAEA Safety Standards Series No. GSR Part 3. IAEA, Vienna. 2014 ISBN: 978-92-0-135310-8.

[3] European Council Directive 2013/59/Euratom on basic safety standards for protection against the dangers arising from exposure to ionising radiation and repealing Directives 89/618/Euratom, 90/641/Euratom, 623 96/29/Euratom, 97/ 43/Euratom and 2003/122/Euratom. Official Journal of the European Union 2014; 57:1-73L13.

[4] ICRP. ICRP publication 135. Diagnostic reference levels in medical imaging. Ann ICRP 2017;46:1-144.

[5] American Association of Physicist in Medicine. The measurement, reporting, and management of radiation dose in CT. Report of AAPM Task Group 23 AAPM report no. 96, 2008 ISBN: 978-1-888340-73-0.

[6] International Atomic Energy Agency. Dosimetry in diagnostic radiology for paediatric patients IAEA human health series No. 24. IAEA, Vienna. 2013 ISBN: 978-92-0-141910-1.

[7] International Commission on Radiation Units and Measurement. Patient Dosimetry for X Rays used in Medical Imaging. ICRU Report 74. 2005 ICRU Bethesda, MD.

[8] Fitousi N. Patient dose monitoring systems: a new way of managing patient dose and quality in the radiology department. Phys Med 2017;44:212-21.

[9] Granata C, Sorantin E, Seuri R, Owens CM. European Society of Paediatric Radiology Computed Tomography and Dose Task Force: European guidelines on diagnostic reference levels for paediatric imaging. Pediatr Radiol 2019;49(5): $702-5$.

[10] Hayton A, Wallace A. Derivation of Australian diagnostic reference levels for paediatric multi detector computed tomography. Australas Phys Eng Sci Med 2016; 39(3):615-26.

[11] Takei Y, Miyazaki O, Matsubara K, Shimada Y, Muramatsu Y, Akahane K, et al. Nationwide survey of radiation exposure during pediatric computed tomography examinations and proposal of age-based diagnostic reference levels for Japan. Pediatr Radiol 2016;46(2):280-5.

[12] Vassileva J, Rehani M, Kostova-Lefterova D, Al-Naemi HM, Al Suwaidi JS, Arandjic D, et al. A study to establish international diagnostic reference levels for paediatric computed tomography. Radiat Prot Dosimetry 2015;165(1-4):70-80.

[13] European Commission. European guidelines on diagnostic reference levels for paediatric imaging. In: Radiation Protection. 185. Luxembourg: European Commission; 2018.
[14] Stamm G, Saure H-D. Entrance surface dose and its correlation with patient parameters. Radiat Prot Dosimetry 1998;80(1-3):235-7.

[15] Kiljunen T, Järvinen H, Savolainen S. Diagnostic reference levels for thorax X-ray examinations of paediatric patients. BJR 2007;80(954):452-9.

[16] Lindskoug BA. The reference man in diagnostic radiology dosimetry. BJR 1992;65 (773):431-7.

[17] Danish Health Authority. Guidance on patient doses and diagnostic reference levels for conventional x-ray examinations of children (in Danish). https://www.sst.dk/d a/Udgivelser/2006/Vejledning-om-patientdoser-og-referencedoser-for-roentge nundersoegelser [accessed 6 June 2021].

[18] Strålsäkerhetscentralens föreskrift om berättigandebedömning och om optimering av strålskyddet vid medicinsk exponering STUK S/4/2019STUK. https://www.st uklex.fi/fi/STUK-S-4-2019-sv_bilaga.pdf [accessed 6 June 2021].

[19] Järvinen H, Seuri R, Kortesniemi M, Lajunen A, Hallinen E, Savikurki-Heikkilä P, et al. Indication-based national diagnostic reference levels for paediatric CT: a new approach with proposed values. Radiat Prot Dosimetry 2015;165(1-4):86-90.

[20] International Atomic Energy Agency. Diagnostic Radiology Physics: A Handbook for Teachers and Students. Vienna: IAEA; 2014.

[21] Leithner R, Homolka P. A quantitative comparison of data evaluation methods to derive diagnostic reference levels for CT from a dosimetric survey: correlation analysis compared to simple evaluation strategies. Physica Med 2013;29:470-7.

[22] Hart D, Wall BF, Shrimpton PC, Dance DR. The establishment of reference doses in paediatric radiology as a function of patient size. Radiat Prot Dosimetry 2000;90 (1):235-8.

[23] De Monte F, Castaldi B, Branchini M, Bettinelli A, Milanesi O, Paiusco M, et al. Typical values for pediatric interventional cardiology catheterizations: a standardized approach towards Diagnostic Reference Level establishment. Phys Med 2020;76:134-41.

[24] Kuczmarski RJ, Ogden CL, Guo SS, et al. 2000 CDC growth charts for the United States: Methods and development. National Center for Health Statistics. Vital Health Stat 11(246).

[25] Spinelli A, Buoncristiano M, Kovacs VA, et al. Prevalence of severe obesity among primary school children in 21 European countries. Obes Facts 2019;12(2):244-58.

[26] American Association of Physicists in Medicine. Size-specific dose estimates (SSDE) in pediatric and adult body CT examinations. Report of AAPM Task group 204. AAPM report no. 204. 2011 ISBN: 978-1-936366-08-8.

[27] American Association of Physicists in Medicine. Use of water equivalent diameter for calculating patient size and size-specific dose estimates (SSE) in CT Report of AAPM Task group 220. 2014 ISBN: 978-1-936366-39-2. 